\title{
BRUSTKREBSFRÜHERKENNUNG
}

\section{Im Alter lieber seltener zur Mammografie}

Bei Frauen über 66 bringt eine jährliche Mammografie keinen größeren Nutzen als eine Mammografie nur alle zwei Jahre. Epidemiologen und Biostatistiker vom Breast Cancer Surveillance Consortium in San Francisco werteten Daten des Konsortiumre-

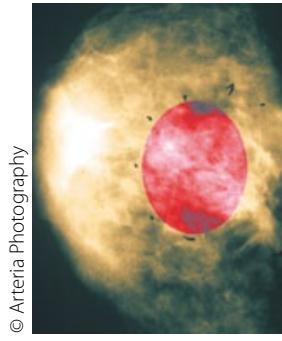

Verdächtig - aber auich maligne? gisters sowie der Krankenversicherung Medicare aus. Die Informationen stammen von fast 300000 Frauen im Alter zwischen 66 und 89 Jahren, die zwischen 1999 und 2006 Mammografien erhalten hatten.
Bei insgesamt knapp 3000 Frauen wurde Brustkrebs diagnostiziert, 1946 von ihnen waren im Jahresabstand und 1047 alle zwei Jahre mammografiert worden. Es zeigte sich keine Assoziation zwischen Komorbidität, Screeningintervall und Tumorstadium zum Zeitpunkt der Diagnose, aber die älteren Frauen waren einem höheren Risiko für falsch-positive Ergebnisse der Mammografie ausgesetzt, wenn die Untersuchung jährlich statt alle zwei Jahre vorgenommen wurde: Beim Ein-Jahres-Rhythmus lag die Falsch-positiv-Rate bei $48 \%$ (95\%-Konfidenzintervall zwischen $46,1 \%$ und $49,9 \%$ ) und bei einer Mammografie nur alle zwei Jahre bei $29 \%$.

I Nat I Cancer Inst. 2013; online 5. Februar. DOI: 10.1093/jnci/djs645

\section{GEWISSENSFRAGE}

\section{Soll man Todkranke noch operieren?}

Soll man todkranke Patienten tatsächlich noch operieren? Viele Ärzte werden da skeptisch reagieren - selbst wenn sicher sein sollte, dass die Betroffenen ohne Operation sterben werden. Anästhesisten aus den USA und Kanada haben jetzt Zahlen geliefert, die auf den ersten Blick nicht gerade ermutigend wirken: Die 30-Tages-Mortalität moribunder Patienten erreicht nach operativen Eingriffen knapp 53\%. „Dabei

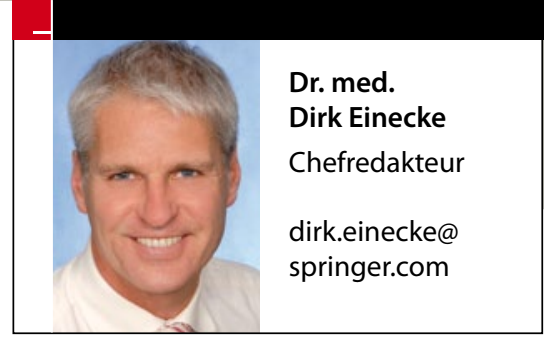

\section{FERTILITÄT}

\section{Fernseher als Spermienkiller}

Drei Stunde Fernsehen pro Tag genügen, um die Spermienzahl zu halbieren, haben Mitarbeiter der Harvard School of Public Health in Boston herausgefunden. Sie bestimmten die Spermienkonzentration und -qualität bei 189 gesunden Männern im Alter von 18 bis 22 Jahren. Es zeigte sich ein deutlicher Zusammenhang zwischen der körperlichen Aktivität und der Spermienproduktion: Die Gesamtzahl der Spermien im Ejakulat war im Quartil der sportlich aktivsten Männer um 41\%, die Spermienkonzentration um $73 \%$ höher als bei den Couch-Potatoes. Männer, die sich gar nicht bewegten und viel fernsahen, kamen auf eine Spermienkonzentration von nur 24 Millionen pro $\mathrm{ml}$, was gerade noch im unteren Normbereich liegt.

Br J Sports Med 2013, online 4. Februar; doi: 10.1136/ bjsports-2012-091644

Eingriff noch.

J Crit Care 2013, online 10. Januar; doi: 0.1016/j. jcrc.2012.10.068

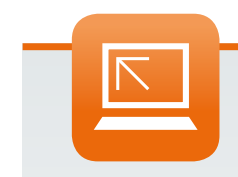

Expertenrat auf springermedizin.de

\section{KNIEGELENKSARTHROSE}

\section{Nimmt Ayurveda die Schmerzen?}

Wenn es bei Kniegelenksarthrose um Analgesie geht, sind ayurvedische pflanzliche Präparate ähnlich wirksam wie Standardarzneien. Dies ergab eine indische, randomisierte Doppelblindstudie an $440 \mathrm{~Pa}-$ tienten mit symptomatischer Kniegelenksarthrose. Je 110 Patienten wurden mit Celecoxib, Glucosaminsulfat bzw. einem von zwei verschiedenen ayurvedischen Ex- trakten sechs Monate lang behandelt. Primäre Endpunkte der Studie waren die Veränderungen der VAS- und WOMAC-Werte für die Schmerzintensität bzw. die Gelenkfunktion. Die Auswertung der Daten ergab keinen Unterschied hinsichtlich der Schmerz- und Funktionsparameter.

Rheumatology 2013, online 30. Januar; doi: 10.1093/ rheumatology/kes414
Guter Rat muss nicht teuer sein. In unserer MMW-Sprechstunde gibt es ihn sogar kostenlos!

Unter www.springermedizin. de/mmw-sprechstunde wissen unsere Experten Prof. FüeßI und Dr. Stiefelhagen Rat bei kniffligen Fragen. 